\title{
ВЛИЯНИЕ ЗАКРУТКИ НА УСТОЙЧИВОСТЬ ТЕЧЕНИЙ В КАНАЛАХ
}

\section{INFLUENS OF SWIRLING ON FLOW STABILITY IN CHANNELS}

\section{Akhmetov}

Summary. The problem of hydrodynamic stability of a swirling flow of a viscous incompressible fluid in a channel with rigid walls is considered. The solution is determined by numerical integration of sixth-order ordinary differential equations system. The amplification coefficients and phase velocities of the most unstable disturbances are calculated for various flow profiles.

Keywords: hydrodynamic stability, swirling flow, viscous fluid, amplification coefficients.

\author{
Ахметов Вадим Каюмович \\ Д.т.н., профессор, ФГБОУ ВО «Начиональный \\ исследовательский Московский государственный \\ строительный университет» \\ vadim.akhmetov@gmail.com
}

Аннотация. Рассматривается задача гидродинамической устойчивости закрученного потока вязкой несжимаемой жидкости в канале с жесткими стенками. Решение определяется численным интегрированием системы обыкновенных дифференциальных уравнений шестого порядка. Рассчитаны коэффициенты усиления и фазовые скорости наиболее неустойчивых возмущений для различных профилей течения.

Ключевые слова: гидродинамическая устойчивость, закрученный поток, вязкая жидкость, коэффициенты усиления.

Базовой системой уравнений гидродинамики вязкой несжимаемой жидкости является система Навье-Стокса, используя которую можно рассчитать основное течение, а затем исследовать его устойчивость. Можно также рассматривать некоторые модельные течения в качестве основного с заданным распределением скоростей.

 \\ и мето $\triangle$ решения}

Будем считать, что основное течение вязкой несжимаемой жидкости является осесимметричным и в цилиндрической системе координат $(r, \varphi, z)$ описывается полем скоростей

$$
V_{z}=U(r), \quad V_{\varphi}=W(r), \quad V_{r}=0
$$

Здесь ось $z$ направлена вдоль движения потока. В классической теории устойчивости на основное течение накладываются возмущения в виде бегущей волны

$$
\begin{aligned}
& \left\{V_{z}^{\prime}, V_{r}^{\prime}, V_{\varphi}^{\prime}, p^{\prime}\right\}= \\
& =\{F, i S, H, P\} \exp [i(\alpha z+n \varphi-\alpha c t)],
\end{aligned}
$$

Комплексные функции $F(r), S(r), H(r), P(r)$ задают амплитуду волны длиной $\lambda$ с волновым числом $\alpha=2 \pi / \lambda$, модой возмущения $n(n=0 ; \pm 1 ; \pm 2 ; \ldots)$ и скоростью распространения $c$. Для неизвестных амплитудных функций после процедуры подстановки возмущений (1) в уравнения Навье-Стокса и ее дальнейшей линеаризации можно получить систему следующих уравнений 

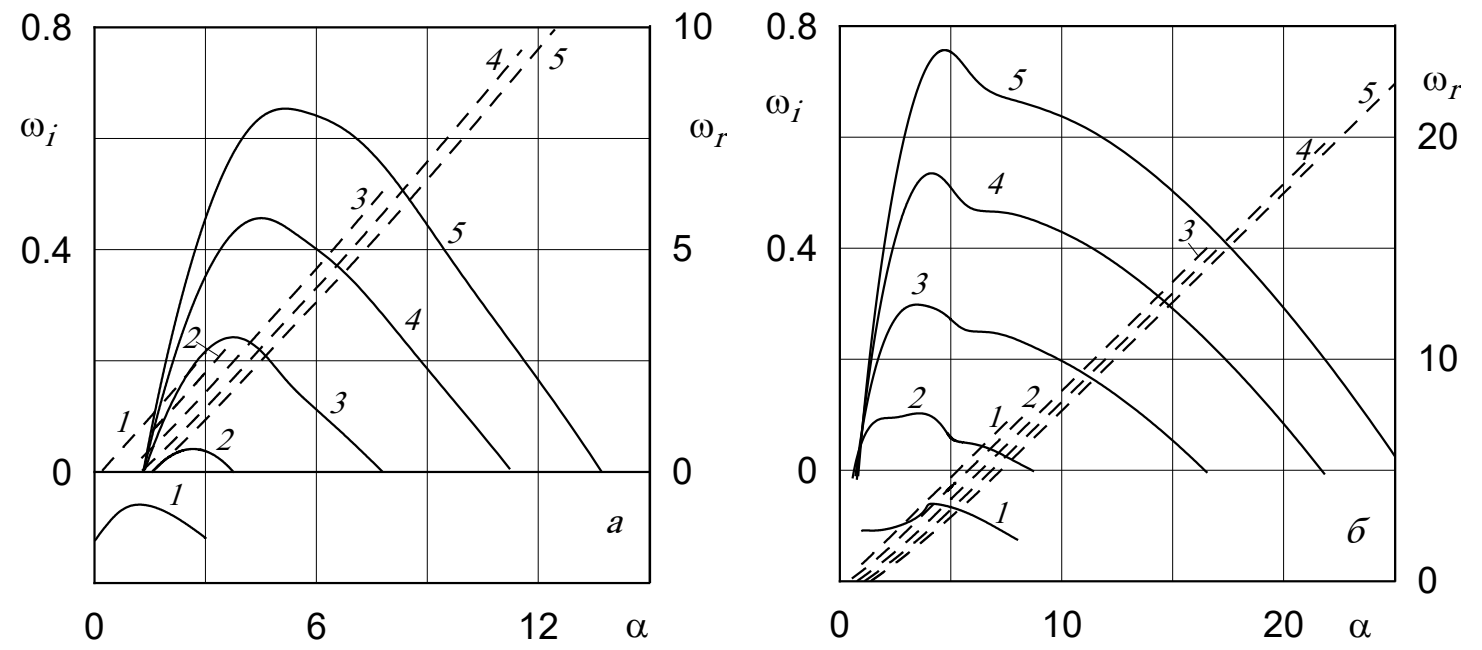

Рис.1. Зависимости коэффициентов усиления (сплошные линии) и частот колебаний (штриховые линии) от волнового числа при : a - $\operatorname{Re}=300,6-\operatorname{Re}=1000,0.1,0.3,0.56,0.8,1$ (кривые 1-5)

$$
\begin{aligned}
& r^{2} \gamma F+\alpha r^{2} P+r^{2} S U^{\prime}=\frac{1}{i \operatorname{Re}}\left[r\left(r F^{\prime}\right)^{\prime}-\left(\alpha^{2} r^{2}+n^{2} F\right)\right] \\
& r^{2} \gamma S+2 r H W-r^{2} P^{\prime}= \\
& =\frac{1}{i \operatorname{Re}}\left[r\left(r G^{\prime}\right)^{\prime}-\left(\alpha^{2} r^{2}+n^{2}+1\right) S-2 n H\right] \\
& r^{2} \gamma H+r^{2} S\left(W^{\prime}+\frac{W}{r}\right)+r n P= \\
& =\frac{1}{i \operatorname{Re}}\left[r\left(r H^{\prime}\right)^{\prime}-\left(\alpha^{2} r^{2}+n^{2}+1\right) H-2 n S\right], \\
& \alpha r F+(r S)^{\prime}+n H=0
\end{aligned}
$$

где $\gamma=\alpha(U-c)+n W / r$. Граничными условиями для системы (3)-(6) служат выражения:

$$
\begin{aligned}
& S(0)=H(0)=0, F(0), P(0)- \\
& \text { ограничены при } n=0 ;
\end{aligned}
$$

$$
\begin{aligned}
& S(0) \pm H(0)=0, F(0)=P(0)=0 \text { - где } n= \pm 1 ; \\
& S(0)=H(0)=F(0)=P(0)=0-\text { где }|n|>1 ; \\
& S\left(r_{k}\right)=H\left(r_{k}\right)=F\left(r_{k}\right)=0 .
\end{aligned}
$$

Будем исследовать, так называемую, временную устойчивость течения (1). При этом волновое число $\alpha$ действительное, а скорость $c=c_{r}+i c_{i}$ комплексная $\left(c_{r}\right.$ - фазовая скорость, $c_{i}$ - скорость роста возмущения по времени). Очевидно, что при $c_{i}<0$ возмущения затухают (течение устойчиво), а при $c_{i}>0$ - растут (течение неустойчиво).
Сформулированная постановка является задачей на собственные значения, которая решается численно интегрированием системы (3)-(6) с условиями (7)-(10) методом Рунге-Кутта с использованием ортогонализационной процедуры Грамма-Шмидта. Метод решения изложен в [11] и применялся при исследовании широкого круга задач.

\section{3. Результаты вычислений}

Рассмотрим результаты исследования устойчивости на примерах течений вида (1) с разными распределениями скорости. В первом случае будем считать, что ядро закрученного потока представляет собой вихрь Бюргерса:

$$
\begin{aligned}
& U(r)=U_{1}(r) / U_{0}, \quad W(r)=W_{1}(r) / U_{0}, \quad U_{0}=U_{1 \max }, \\
& U_{1}(r)=U_{2}\{1-\exp [\tau(r-1)]\}+\varepsilon\left[\exp \left(-\sigma r^{2}\right)-\exp (-\sigma)\right] \\
& W_{1}(r)=W_{2}\{\exp (-\tau)-\exp [\tau(r-1)]\}+ \\
& +\frac{\beta}{r}\left[1-\exp \left(-\sigma r^{2}\right)\right], \\
& U_{2}=\frac{\tau^{2}}{\sigma} \frac{\sigma-\varepsilon[1-(1+\sigma) \exp (-\sigma)]}{\tau^{2}+2[1-\sigma-\exp (-\sigma)]}, \quad W_{2}=\beta \frac{1-\exp (-\sigma)}{1-\exp (-\tau)}
\end{aligned}
$$

Здесь параметр $\tau$ определяет толщину пограничного слоя, $\varepsilon$ задает форму профиля ( $\varepsilon>0-$ струйное течение, $\varepsilon<0-$ спутное, $\varepsilon=0$ - равномерный поток).

Выборочные результаты устойчивости течения (11) при $\varepsilon=0, \tau=12, \sigma=18, n=-1$ представлены на рис. 1. Небольшая закрутка $(\beta=0.1)$ не оказывает существенного воздействия на поток и он остается устойчивым (кри- 

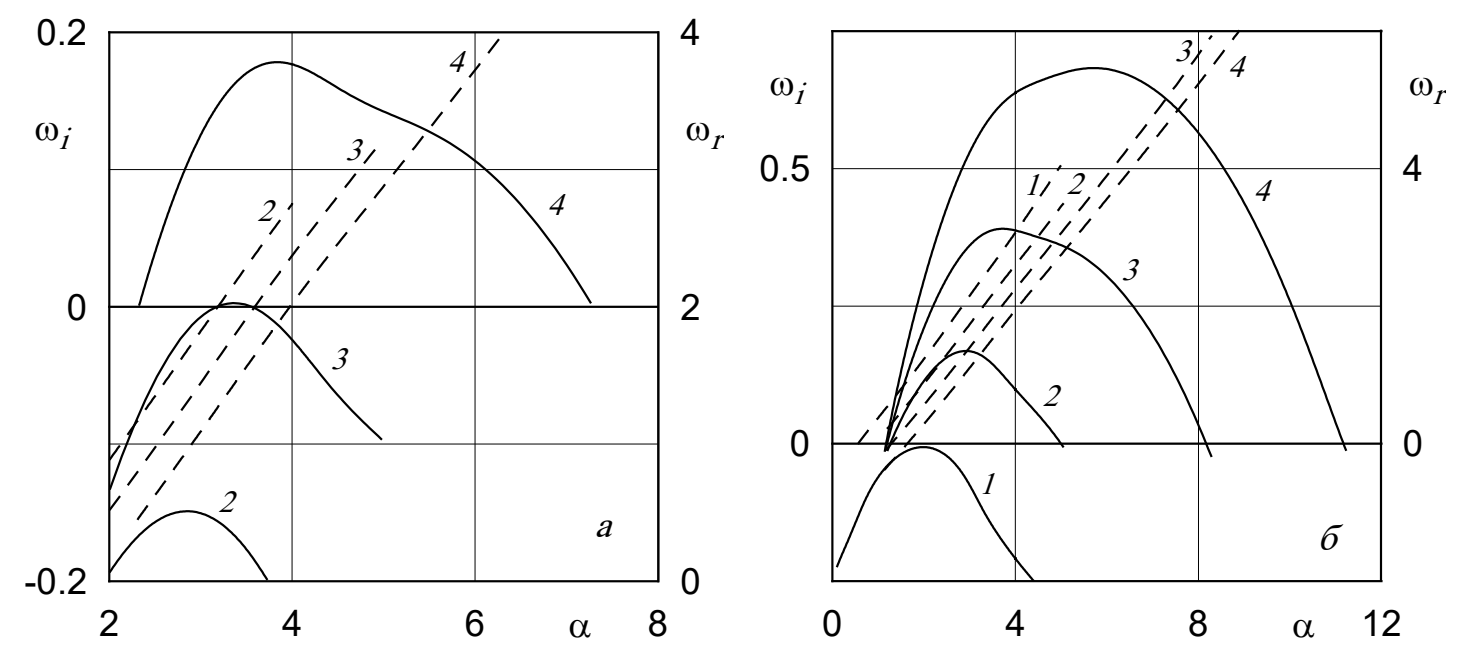

Рис. 2. Зависимости коэффициентов усиления (сплошные линии) и частот колебаний (штриховые линии) от волнового числа при: $\mathrm{a}-\mathrm{Re}=100,6-300, \mathrm{G}=0.7,1.2,1,7,2.2$ (кривые 1-4)

вая 1). При увеличении закрутки $(\beta=0.3)$ устойчивость потока нарушается, а в дальнейшем - усиливается. Длина волны, которая соответствует наиболее неустойчивым возмущениям, примерно равна диаметру канала и незначительно уменьшается с увеличением закрутки. Данный профиль скорости неустойчив к более коротким возмущениям по сравнению с течением Пуазейля во вращающейся трубе. Рассмотренные моды неустойчивости имеют невязкий характер и с ростом значения числа Рейнольдса неустойчивость сохраняется.

Во втором случае рассмотрим течение с полем скоростей вида:

$$
\begin{aligned}
& V_{z 0}(r)=D_{0}+D_{1} \exp \left(-B_{1} r^{2}\right) \\
& V_{\varphi 0}(r)=G \frac{A}{r}\left(1-\exp \left(-B_{2} r^{2}\right), 0 \leq r \leq r_{0}\right. \\
& V_{z 0}(r)=a_{0}+a_{1} r+a_{2} r^{2}, V_{\varphi 0}(r)=G\left(b_{0}+b_{1} r+b_{2} r^{2}\right) \\
& r_{0} \leq r \leq 1 .
\end{aligned}
$$

\section{В данном исследовании примем}

$D_{0}=1, \quad D_{1}=0, \quad B_{1}=0, \quad B_{2}=14, \quad A=0.419$, $r_{0}=0.75, a_{0}=-8, a_{1}=24, a_{2}=-16$, $b_{0}=-5.363, b_{1}=19.627, b_{2}=-14.260$.
Дисперсионные зависимости, характеризующие устойчивость течения (12) при $R e=100,300$ представлены на рис. 2. В этом случае малая закрутка $G=0.7$, 1.2 также не приводит к нарушению устойчивости потока, а при усилении закрутки $G=1.7,2.2$ поток становится неустойчивым. Такая же картина наблюдается при больших значениях чисел Рейнольдса. Как и в предыдущем случае, длина волны для наиболее неустойчивых возмущений имеет значение порядка длины канала.

\section{4. Эак^ючение.}

Для течений во вращающемся канале с постоянной угловой скоростью $q$ при превышении критического числа Рейнольдса нарушение устойчивости наблюдается при чрезвычайно малых значениях $q \approx 0.01$. Представленные вычисления течений с закруткой, распределенной по радиусу, показывают существование некоторой критической закрутки. Определяя параметр закрутки $G$ отношением максимальных значений азимутальной компоненты скорости к осевой, для представленных вычислений в диапазоне умеренных чисел Рейнольдса критическое значение закрутки составляет $G_{\kappa p} \approx 0.7-1.7$.

Работа выполнена при финансовой поддержке РФФИ (№ 18-01-00762).

\section{ЛИТЕРАТУРА}

1. Гупта А., Лилли Д., Сайред Н. Закрученные потоки. М.: Мир, 1987. 588 с.

2. Карелин В.Я., Кривченко Г.И., Мордасов А.П., Волшаник В.В., Зуйков А.Л., Ахметов В.К. Физическое и математическое моделирование систем гашения энергии в вихревых водосбросах // Физическое и математическое моделирование гидравлических процессов при исследовании гидроузлов комплексного назначения. Тез. научно-техн. совещания (г. Дивногорск, 24-26 мая 1989). Л.: ВНИИГ им. Б.Е. Веденеева, 1989. С. 11-12. 
3. Rukes L., Sieber M., Paschereit C., Oberleithner K. The impact of heating the breakdown bubble on the global mode of a swirling jet: experiments and linear stability analysis // Physics of Fluids. 2016. Vol. 28. 104102.

4. Ахметов В.К., Волшаник В.В. Исследование распространения аэрированной затопленной струи // Гидротехническое строительство. 1994. № 10. С. 2426.

5. Ахметов В.К., Шкадов В.Я., Шкадова В.П. Смешение нагретых газов в осесимметричном канале с предварительной закруткой потока // Известия РАН. Механика жидкости и газа. 2006. № 3. С. 19-29.

6. Ахметов В.К., Шкадов В.Я. Распыление порошка закрученным потоком с зоной рециркуляции // Известия РАН. Механика жидкости и газа. 2000. № 6. C. 3-15.

7. Ахметов В.К., Шкадов В.Я. К вопросу об устойчивости свободного вихря // Вестник МГУ. Сер. 1. Математика, механика. 1987. № 2. С. 35-40.

8. Chan S.; Ault J.T., Haward S.J., Meiburg E., Shen A.Q. Coupling of vortex breakdown and stability in a swirling flow // Physical rewiew fluids. 2019. V. 4. № 8. 084701.

9. Blanco-Rodríguez F.J., Rodríguez-García J.0., Parras L., del Pino C. Optimal response of Batchelor vortex // Physics of Fluids. 2017. Vol. 29. 064108.

10. Yadav N.K., Samanta A. The stability of compressible swirling pipe flows with density stratification // Journal of Fluid Mechanics. 2017. Vol. 823. Pp. 689-715.

11. Ахметов В.К. Гидродинамическая устойчивость контрвихревых течений // Гидротехническое строительство. 2018. № 2. С. 13-18.

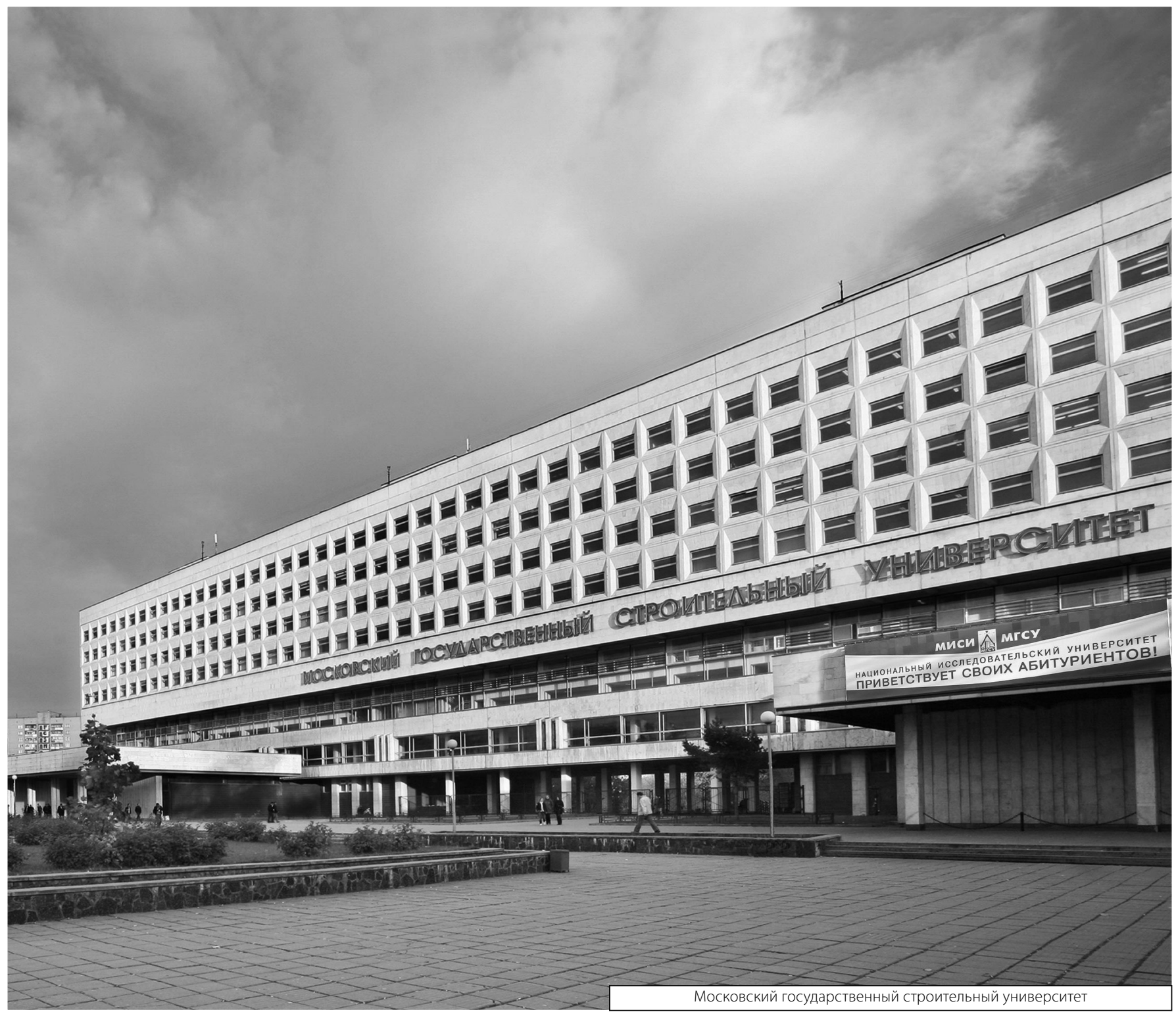

